\section{RMD Open}

Rheumatic \&

Musculoskeletal Diseases

\title{
Assessment of the Patient Acceptable Symptom State (PASS) in psoriatic arthritis: association with disease activity and quality of life indices
}

\author{
Ennio Lubrano (1D , ${ }^{1}$ Silvia Scriffignano, ${ }^{1}$ Ana Belen Azuaga, ${ }^{2}$ Julio Ramirez, ${ }^{2}$ \\ Juan D Cañete, ${ }^{3}$ Fabio Massimo Perrotta ${ }^{1}$
}

To cite: Lubrano $\mathrm{E}$, Scriffignano S, Azuaga AB, et al. Assessment of the Patient Acceptable Symptom State (PASS) in psoriatic arthritis: association with disease activity and quality of life indices. RMD Open 2020;6:e001170. doi:10.1136/ rmdopen-2020-001170

Received 2 January 2020 Revised 12 February 2020 Accepted 22 February 2020

Check for updates

(C) Author(s) (or their employer(s)) 2020. Re-use permitted under CC BY-NC. No commercial re-use. See rights and permissions. Published by BMJ.

${ }^{1}$ Dipartimento di Medicina e Scienze della Salute 'Vincenzo Tiberio', Università degli Studi del Molise, Campobasso, Italy ${ }^{2}$ Hospital Clinic de Barcelona, Barcelona, Catalunya, Spain ${ }^{3}$ Unidad de Artritis Seervicio de Reumologia, Hospital Clinic de Barcelona, Barcelona, Catalunya, Spain

Correspondence to Professor Ennio Lubrano; enniolubrano@hotmail.com

\section{ABSTRACT}

Objective The aim of this study was to evaluate the discriminant capability of the Patient Acceptable Symptom State (PASS) according to disease activity, remission/ low disease activity indices and quality of life indices in patients with psoriatic arthritis (PSA)

Methods Consecutive patients with PsA were enrolled in this cross-sectional study. At each visit, the patients underwent a complete physical examination and their clinical/laboratory data were collected. Disease activity was assessed using the Disease Activity Score for Psoriatic Arthritis (DAPSA) and remission/low disease activity using the DAPSA minimal disease activity (MDA) and very low disease activity (VLDA) criteria. The Psoriatic Arthritis Impact of Disease (PsAID) and the Health Assessment Questionnaire-Disability Index scores were also collected. Finally, PASS was assessed by asking all patients to answer yes or no to a single question.

Results Patients who answered yes to PASS showed a significantly better overall mean DAPSA score than those who were not in PASS. Furthermore, patients in PASS showed a significantly lower level of systemic inflammation, lower Leeds Enthesitis Index score, a significantly lower impact of disease (PSAID), lower pain and better function than patients who answered no to PASS. A moderate to good agreement was found between PASS, MDA, DAPSA low disease activity and PSAID score $\leq 4$. Good sensitivity and specificity were found with PASS with respect to DAPSA low disease activity, and although PASS is sensitive in the identification of patients with MDA, DAPSA remission and VLDA it lacks of specificity.

Discussion This study showed that PASS might be used as an alternative to determine disease activity in patients with PsA in real clinical practice, mainly in patients with low disease activity according to DAPSA criteria.

\section{INTRODUCTION}

Psoriatic arthritis (PsA) is a complex and chronic inflammatory disease characterised by an association between psoriasis and arthritis. It could be recognised as a 'syndrome', where different manifestations such as psoriasis, peripheral and axial joint

\section{Key messages}

What is already known about this subject?

- In daily clinical practice, evaluation of disease activity is of crucial importance in the management of patients with psoriatic arthritis, and sharing treatment decisions is not always feasible because it is time-consuming and also because of lack of data.

What does this study add?

- The main result of this study is that the Patient Acceptable Symptom State showed good sensitivity and specificity in determining a condition of low disease activity according to the Disease Activity Score for Psoriatic Arthritis, with the possibility of judging patients in low disease activity using a single simple question.

How might this impact on clinical practice?

- Our results could help clinicians in the assessment of psoriatic arthritis, providing some practical insights.

involvement, enthesitis, extra-articular manifestations, and comorbidities can present at the same time or during the disease course. ${ }^{1}$ The achievement of the best possible disease control such as disease remission or low disease activity has been proposed as a treatment target and may be an achievable goal for patients with PsA. ${ }^{2-5}$ Thus, due to the complexity of the disease, unidimensional and multidimensional disease activity indices that include the assessment of all disease domains, or that are mainly focused on joint involvement, were developed. This could lead to a discrepancy in the assessment of disease activity, with the possibility to judge a patient in remission/low disease activity or not by different indices used. ${ }^{6}$ Finally, due to the construction of these indices, residual disease activity could persist, mainly in patients with PsA who achieve less stringent criteria such 
as low disease activity, with the possibility of the patient being in remission in one domain (joints), but with active disease in other domains (eg, skin or enthesis) ${ }^{7}$ In daily life, the need to change the therapeutic regimen can be decided by the physician or shared with the patient, even if often patients' thoughts and perspectives are not completely considered. Thus, in the last few years, there has been a shift towards a more patient-centred perspective with regard to the disease, adopting patient-reported outcomes which reflect patients' perceptions on their health status or treatment and are reported by the patients themselves. ${ }^{89}$ Furthermore, in daily clinical practice, evaluation of disease activity is not always feasible because it is time-consuming and because of lack of data. ${ }^{10}$ In this context, we previously demonstrated that a simple instrument such as the Patient Global Assessment (PtGA) correlates with disease activity indices and could be potentially used as an alternative for evaluating global disease activity. ${ }^{11}$ More recently, the Outcome Measures in Rheumatology Clinical Trials has recommended the measurement of patients' well-being according to a dichotomous condition: satisfactory versus unsatisfactory status. ${ }^{12}$ In this context, the Patient Acceptable Symptom State (PASS), a single-question tool for evaluating the level of symptoms at which patients consider themselves well, is another instrument that could be adopted. ${ }^{13}$ PASS has previously been evaluated in patients affected by ankylosing spondylitis, osteoarthritis, rheumatoid arthritis and systemic lupus erythematosus, and has a significant association with disease activity. ${ }^{14-18}$ However, few data are available on the possible application of PASS in patients affected by PsA, ${ }^{19}$ and the possibility of using a simple question that may encompass disease status in different and complex domains could be intriguing. Our study was aimed to take into account PASS as a simple and useful instrument to picture and judge overall disease status in real clinical setting. Thus, the objective of this study was to assess the discriminant capability, agreement, sensitivity and specificity of PASS compared with disease activity and remission indices (Disease Activity Score for Psoriatic Arthritis (DAPSA) ${ }^{20}$ minimal disease activity (MDA) and very low disease activity (VLDA) $)^{21} 22$ and with measures of function and quality of life (Psoriatic Arthritis Impact of Disease (PsAID) and Health Assessment Questionnaire-Disability Index (HAQ-DI) $)^{23} 24$ in patients with PsA.

\section{PATIENTS AND METHODS}

\section{Patient selection}

In this cross-sectional study, patients were enrolled at the Rheumatology Unit, Department of Medicine and Health Science, University of Molise, Italy, and at Servicio de Reumatología, Hospital Clínic de Barcelona, Spain. During the study period (from 1 January 2019 to 30 September 2019), all patients with PsA who were on at least 6 months of follow-up treatment with conventional and/or biologic disease-modifying antirheumatic drugs were considered potentially eligible for the study.

The following were the inclusion criteria:

- PsA classified with the CASPAR (ClASsification criteria for Psoriatic ARthritis) criteria. ${ }^{25}$

- Age $\geq 18$ years.

- At least 6 months of follow-up at the study visit (patients had to have been treated for at least 6 months in our centres).

\section{Data collection}

Detailed medical history and physical examination were performed in all patients. Demographics and disease characteristics including gender, age, disease duration, level of education and pattern of articular manifestations were carefully collected. Laboratory parameters were also evaluated. Clinical assessment encompassed the number of tender joints (of the 68 assessed joints) and swollen joints (total of 66 joints), enthesitis and dactylitis. Enthesitis was assessed using the Leeds Enthesitis Index (LEI) ${ }^{26}$ and dactylitis assessed as present or absent, as published elsewhere. ${ }^{27}$ Skin assessment was performed using the body surface area (BSA). The HAQ-DI, ${ }^{24}$ PtGA and pain assessment on Visual Analogue Scale (VAS $1-10 \mathrm{~cm})$ were performed by all patients. Physician's global evaluation of disease activity on a VAS scale was also recorded. ${ }^{11}$

\section{Disease activity indices and remission/low disease activity indices (MDA, VLDA and DAPSA)}

MDA was defined according to Coates $e t a l^{21}$ Patients were considered in MDA when they satisfied five of the following seven criteria: tender joint count $\leq 1$; swollen joint count $\leq 1$; BSA $\leq 3 \%$; VAS pain score of $\leq 15$; PtGA VAS score of $\leq 20$; HAQ-DI score $\leq 0.5$; and tender entheseal points $\leq 1$.

Patients were considered in VLDA when all seven criteria were met. ${ }^{22}$ DAPSA score was calculated by adding the number of tender and swollen joints, VAS pain, PtGA and $\mathrm{C}$ reactive protein $(\mathrm{CRP})(\mathrm{mg} / \mathrm{dL}) .{ }^{20}$ A DAPSA score of $\leq 4$ means disease remission, while a DAPSA score of $\leq 14$ means a condition of low disease activity.

\section{Function and quality of life indices (HAQ-DI and PsAID)}

The HAQ-DI and the PsAID were evaluated as measures of function and quality of life. In particular we used the 12-item version of the PsAID. ${ }^{23} \mathrm{HAQ}-\mathrm{DI} \leq 0.5$ was defined as good functional status and PsAID $\leq 4$ as low disease impact.

\section{PASS assessment}

The global question assessing PASS was formulated as the following: 'Think about all the ways your PsA has affected you during the last 48 hours. If you were to remain in the next few months as you were during the last 48 hours, would this be acceptable to you?' The yes/no response was used as an external indicator of the patient's satisfaction (ie, PASS) with their current symptoms state. 


\section{Statistical analysis}

Statistical analysis was performed using the SPSS V.17 package and GraphPad Prism V.5. Normally distributed variables were summarised using mean $\pm \mathrm{SD}$, and non-normally distributed variables by median and IQR. Patients reporting an acceptable symptoms state (PASS-yes) were compared with patients reporting an unacceptable symptoms state (PASS-no) with regard to demographics and disease characteristics by descriptive statistics. Mann-Whitney test was performed accordingly. Univariate comparisons between nominal variables were calculated using $\chi^{2}$ test or Fisher's test where appropriate. The agreement between PASS-yes and the different indices used to assess remission/low disease activity was carried out through Cohen's kappa test (kappa <0: no agreement; between 0.00 and 0.20 : slight agreement; between 0.21 and 0.40: fair agreement; between 0.41 and 0.60: moderate agreement; between 0.61 and 0.80 : good agreement; and between 0.81 and 1.00: almost perfect agreement).$^{28}$ Moreover, sensitivity, specificity, positive predictive value, negative predictive value and likelihood ratio of PASS-yes with respect to the different indices used were calculated. To identify the cut-off related to DAPSA, PsAID and HAQ-DI with respect to PASS, we used the receiver operating characteristics (ROC) curve and calculated the sensitivity, specificity and area under the curve (AUC, 95\% CI) of the different outcome measures with respect to PASS. Two-tailed $\mathrm{p}$ values were reported, and $p$ values less than 0.05 were considered significant.

\section{RESULTS}

A total of 118 patients with PsA who met the inclusion criteria were consecutively recruited. Table 1 summarises patients' demographics and disease characteristics. Of these, $79(66.9 \%)$ were male, and $36.8 \%$ came from Spain and $63.2 \%$ from Italy. The mean (SD) age was 56.5 (13.2) years, and the median (IQR) disease duration was $10(3-10)$ years. The median DAPSA score was 9.3. There were $80(67.7 \%)$ patients who reported an acceptable symptoms state (PASS-yes). No patients refused to answer the question, suggesting the acceptability of PASS. No patients asked the examiner to repeat the question due to non-comprehension, and there was no difference in the percentage of patients answering yes or no according to the level of education.

\section{Discriminative capability}

Table 2 compares the demographics and disease characteristics between patients with PsA who reported an acceptable symptoms state (PASS-yes) and patients with an unacceptable symptoms state (PASS-no). Patients who reported PASS-yes showed a significantly better overall mean DAPSA score than those who reported PASS-no. Furthermore, patients who reported PASS-yes showed a significantly lower level of systemic inflammation (CRP), lower LEI, significantly lower impact of disease (PsAID), lower pain and better function than patients who
Table 1 Demographic and clinical disease activity characteristics of patients with PsA

\begin{tabular}{ll}
\hline Female/male & $39 / 79$ \\
\hline Mean (SD) age, years & $56.5(13.2)$ \\
\hline BMI, median (IQR) & $27(25-30.5)$ \\
\hline Disease duration, median (IQR), years & $10(3-17)$ \\
\hline Axial involvement, $\mathrm{n}(\%)$ & $21(17.7)$ \\
\hline Tender joints, median (IQR) & $2(0-4)$ \\
\hline Swollen joints, median (IQR) & $1(0-1)$ \\
\hline BSA, \% (IQR) & $2(0-4)$ \\
\hline Enthesitis (LEI), median (IQR) & $0(0-1)$ \\
\hline CRP, median (IQR), mg/dL & $0.4(0.2-0.6)$ \\
\hline MDA 5/7, n (\%) & $57(48.3)$ \\
\hline DAPSA, median (IQR) & $9.3(4-16.2)$ \\
\hline HAQ-DI, median (IQR) & $0.25(0.125-0.5)$ \\
\hline PsAID (IQR) & $2.2(1-4.2)$ \\
\hline VAS pain, median (IQR), cm & $4(1-6)$ \\
\hline PtGA, median (IQR), cm & $3(1.5-6)$ \\
\hline PASS yes/no & $80 / 38$ \\
\hline Treatment, n (\%) & \\
\hline csDMARD monotherapy & $33(27.9)$ \\
\hline Etanercept & $20(16.9)$ \\
\hline Adalimumab & $19(16.2)$ \\
\hline Golimumab & $6(5)$ \\
\hline Ustekinumab & $16(13.6)$ \\
\hline Secukinumab & $16(13.6)$ \\
\hline Ixekizumab & $5(4.3)$ \\
\hline Apremilast & $3(2.5)$ \\
\hline BMI, body mas & \\
\hline
\end{tabular}

BMI, body mass index; CRP, C reactive protein; csDMARD, conventional synthetic disease-modifying antirheumatic drugs; DAPSA, Disease Activity Score for Psoriatic Arthritis; HAQ-DI, Health Assessment Questionnaire-Disability Index; MDA, minimal disease activity; PASS, Patient Acceptable Symptom State; PsA, psoriatic arthritis; PsAID, Psoriatic Arthritis Impact of Disease; PtGA, Patient Global Assessment; VAS, Visual Analogue Scale.

reported PASS-no. A significantly lower BSA was found in patients who reported PASS-yes; however, in our group, the median BSA was overall low. Finally, $87.5 \%$ of the 80 patients who reported PASS-yes were in treatment with biologic drugs (including apremilast), with respect to $71 \%$ of the 38 patients who reported PASS-no. However, this difference was not statistically significant $(\mathrm{p}=0.13)$.

\section{Performance in the identification of remission/low disease activity}

To assess the performance of PASS in the identification of remission/low disease activity, we evaluated its agreement with MDA, VLDA and DAPSA remission/ low disease activity (table 3). Furthermore, to assess the performance of PASS in the identification of good functional status and low impact of disease, we evaluated its agreement with HAQ-DI and PsAID. A moderate to 
Table 2 Comparison of patients with PsA who reported PASS-yes and PASS-no in the different disease domains (t-test or Mann-Whitney test for unpaired samples) (quantitative variables)

\begin{tabular}{llcc}
\hline & PASS-yes & PASS-no & P value \\
\hline Age, mean (SD) & $57.4(13.4)$ & $53.2(12.9)$ & 0.13 \\
Disease duration, median (IQR) & $10(4.2-15.7)$ & $10(3.75-19.25)$ & 0.99 \\
CRP, mg/dL, median (IQR) & $0.4(0.2-0.55)$ & $0.5(0.4-0.88)$ & 0.04 \\
DAPSA, median (IQR) & $6.4(4-13.5)$ & $18.8(15.34-24.6)$ & $<0.001$ \\
LEI, median (IQR) & $0(0-0)$ & $0(0-1.5)$ & 0.01 \\
BSA, \%, median (IQR) & $0.9(0-3)$ & $1(1-10)$ & 0.001 \\
HAQ-DI, median (IQR) & $0.13(0-0.5)$ & $1(0.5-1.4)$ & $<0.001$ \\
PsAID & $1.7(0.7-3.2)$ & $4.9(3.3-6.8)$ & $<0.001$ \\
VAS pain & $3(1-5)$ & $6(4-8)$ & $<0.001$ \\
PtGA & $3(1-5)$ & $6(5-8)$ & $<0.001$ \\
BMI & $26.7(23-29.3)$ & $27.1(23.8-30.3)$ & 0.55 \\
\hline
\end{tabular}

BMI, body mass index; BSA, body surface area; CRP, C reactive protein; DAPSA, Disease Activity Score for Psoriatic Arthritis; HAQ-DI, Health Assessment Questionnaire-Disability Index; LEI, Leeds Enthesitis Index; PASS, Patient Acceptable Symptom State; PsA, psoriatic arthritis; PsAID, Psoriatic Arthritis Impact of Disease; PtGA, Patient Global Assessment; VAS, Visual Analogue Scale.

good agreement was found between PASS, MDA, DAPSA low disease activity and PsAID score $\leq 4$, while no to fair agreement was found with VLDA and DAPSA remission. Table 4 shows the sensitivity, specificity, positive predictive value, negative predictive value and likelihood ratio of PASS-yes for the presence of a condition of MDA, VLDA, DAPSA low disease activity, DAPSA remission, PsAID $\leq 4$ and HAQ-DI $\leq 0.5$. Interestingly, good sensitivity and specificity were found for PASS-yes with respect to a condition of low disease activity according to DAPSA and low impact of disease according to PsAID, while high sensitivity but low specificity were demonstrated with respect to MDA.

Finally, to assess the cut-off values of the different disease activity, function and quality of life indices with respect to PASS, ROC curves were elaborated. Interestingly, a DAPSA score of 14.8 best differentiated patients with PsA who reported PASS-yes from those who reported PASS-no, with an AUC of 0.83 (95\% CI 0.73 to 0.93), sensitivity of $84 \%$ and specificity of $78.4 \%$. A PsAID score of 4 best differentiated patients with PsA who reported PASS-yes from those who reported PASS-no, with an AUC of 0.82 (95\% CI 0.72 to 0.92 ), sensitivity of $75 \%$ and specificity of $86 \%$, while an HAQ-DI score of 0.62 best differentiated patients with PsA who reported PASS-yes from those who reported PASS-no, with an AUC of $0.78(95 \%$ CI 0.66 to 0.9 ), sensitivity of $75 \%$ and specificity of $81 \%$ (figure 1).

\section{DISCUSSION}

PsA is a complex and multifaceted disease with the possible involvement of different domains. To evaluate disease activity and remission, several unidimensional and multidimensional indices have been proposed. ${ }^{6}$ However, great heterogeneity was reported. Furthermore, some disease activity indices could not be easily used for assessment in routine clinical practice since they are time-consuming and incorporate questionnaires (Short Form-36 (SF-36), HAQ) that are not easy to perform in real-life setting. Finally, frequent use of structured disease activity measures would be beneficial for assessment of inflammatory arthritis, but it remains unclear if rheumatologists adopt them consistently. ${ }^{29}$

PASS proved to be a simple and reliable instrument for assessment of disease status in several rheumatic conditions (ankylosing spondylitis, osteoarthritis, rheumatoid arthritis, systemic lupus erythematosus) and even in PsA. ${ }^{13-19} 30$ PASS is a very quick test which, if administered correctly, can reflect the global status of patients, simplifying some routine assessments. In PsA, it may reflect a simple overall evaluation of patients. In our study, we found that PASS can discriminate patients with respect to disease activity in all disease domains. Interestingly, no significant differences in body mass index (BMI) were found between patients who reported PASS-yes and those who reported PASS-no, with the possibility of patients

\begin{tabular}{|c|c|c|c|c|c|c|}
\hline & DAPSA LDA & DAPSA remission & MDA & VLDA & PsAID $\leq 4$ & HAQ-DI $\leq 0.5$ \\
\hline PASS & $0.72(0.08)$ & $0.22(0.06)$ & $0.45(0.07)$ & $0.21(0.06)$ & $0.51(0.11)$ & $0.44(0.12)$ \\
\hline
\end{tabular}

DAPSA, Disease Activity score for Psoriatic Arthritis; HAQ-DI, Health Assessment Questionnaire-Disability Index; LDA, low disease activity; MDA, Minimal Disease Activity; PASS, patient's acceptable symptoms scale; PsAID, psoriatic arthritis impact of disease; VLDA, Very Low Disease Activity. 
Table 4 Sensitivity, specificity, positive predictive value, negative predictive value and likelihood ratio of PASS with respect to the different indices used

\begin{tabular}{llllll}
\hline & $\begin{array}{l}\text { Sensitivity } \\
\text { (95\% Cl) }\end{array}$ & $\begin{array}{l}\text { Specificity } \\
\mathbf{( 9 5 \% ~ C l )}\end{array}$ & $\begin{array}{l}\text { Positive predictive } \\
\text { value }(\mathbf{9 5 \%} \mathrm{Cl})\end{array}$ & $\begin{array}{l}\text { Negative } \\
\text { predictive } \\
\text { value }(\mathbf{9 5 \%} \mathrm{Cl})\end{array}$ & $\begin{array}{l}\text { Likelihood } \\
\text { ratio }\end{array}$ \\
\hline MDA & $0.96(0.87$ to 0.99$)$ & $0.46(0.32$ to 0.61$)$ & $0.67(0.55$ to 0.77$)$ & $0.92(0.73$ to 0.99$)$ & 1.8 \\
\hline VLDA & $1(0.82$ to 1$)$ & $0.35(0.22$ to 0.48$)$ & $0.30(0.18$ to 0.44$)$ & $1(0.80$ to 1$)$ & 1.6 \\
\hline $\begin{array}{l}\text { DAPSA low disease } \\
\text { activity }\end{array}$ & $0.81(0.66$ to 0.91$)$ & $1(0.81$ to 1$)$ & $1(0.9$ to 1$)$ & $0.69(0.48$ to 0.85$)$ & - \\
\hline DAPSA remission & $1(0.76$ to 1$)$ & $0.38(0.24$ to 0.53$)$ & $0.32(0.19$ to 0.48$)$ & $1(0.81$ to 1$)$ & 1.62 \\
\hline PSAID $\leq 4$ & $0.79(0.65$ to 0.89$)$ & $0.77(0.52$ to 0.93$)$ & $0.90(0.77$ to 0.97$)$ & $0.58(0.36$ to 0.77$)$ & 3.5 \\
\hline HAQ-DI $\leq 0.5$ & $0.86(0.74$ to 0.93$)$ & $0.68(0.43$ to 0.87$)$ & $0.89(0.78$ to 0.95$)$ & $0.61(0.38$ to 0.81$)$ & 2.7 \\
\hline
\end{tabular}

DPASA, disease activity score for psoriatic arthritis; HAQ-DI, Health Assessment Questionnaire-Disability Index; MDA, minimal disease activity; PASS, Patient's acceptable symptom state; PSAID, Psoriatic arthritis impact of disease; VLDA, very low disease activity.

with high BMI achieving a status of good disease control. Furthermore, PASS had, respectively, a moderate to good concordance with both the MDA criteria and the DAPSA criteria for low disease activity. In keeping with this result, PASS also demonstrated good sensitivity and specificity with respect to DAPSA low disease activity, PsAID $\leq 4$ and HAQ-DI $\leq 0.5$, suggesting PASS is a useful and simple

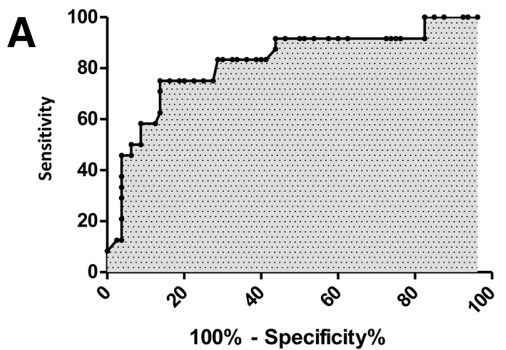

AREA: 0.82 $95 \%$ CI: 0.72 to 0.92

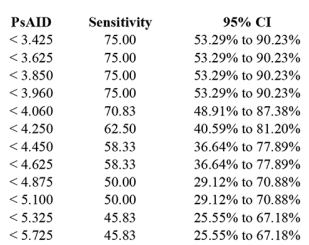

alternative for assessment of not only a low disease state, but also of the low impact of the disease on quality of life and function in patients with PsA. The results of our study confirm what has been reported in a recent study published by Fei et $a l{ }^{19}$ where the authors identified the PASS thresholds for the Psoriatic Arthritis Disease Activity Score (PASDAS) and DAPSA. They found that

Figure 1 Results of the ROC curve analysis for the (A) PsAID threshold for PASS, (B) DAPSA threshold for PASS and (C) HAQ-DI threshold for PASS. DAPSA, Disease Activity Score for Psoriatic Arthritis; HAQ-DI, Health Assessment QuestionnaireDisability Index; PASS, Patient Acceptable Symptom State; PsAID, Psoriatic Arthritis Impact of Disease; ROC, receiver operating characteristics. 
the PASS threshold for PASDAS was in the moderate disease activity range, while the threshold for DAPSA was in the low disease activity range, a result very similar to that found in our study.

Although PASS captures many domains of disease activity and status of PsA, it is obviously not considered so comprehensive as to replace all of the process and outcome measures, as well as the objective signs of inflammation (ie, CRP). In fact, it is a single-item measurement with limited face validity. However, our results suggest that PASS can be deemed a potential alternative for assessment of disease status from the patient's perspective. Obviously, the low concordance found with more stringent disease activity measures could be explained by the intrinsic nature of the PASS question, in which an 'acceptable' status does not completely fit the concept of remission.

There are some limitations to the present study, including its small sample size, its cross-sectional design, and the sensitivity of PASS to change which was not evaluated. Indeed, the present study only indicates some degree of agreement with composite clinical endpoints, well accepted, but not associated with long-term outcomes such as radiographic outcomes. Thus, further studies may be required, mainly to evaluate the performance of PASS in long-term follow-up. A challenge with using PASS in PsA is that it may change with disease course: disease manifestations may change and PASS is known to change over time. We do believe that a multifaceted and complex disease such as PsA could affect the judgement of patients during their natural disease course. Further study to assess the reliability and sensitivity to change should be performed.

Finally, our study demonstrated that PASS is in keeping with a condition of low disease activity but not with MDA or remission. In fact, the more stringent the outcome measure, the lower its specificity. Moreover, the good sensitivity and specificity of PASS with respect to PsAID can be explained by the fact that both instruments are based on 'subjective' domains more than 'objective' ones, underlining that both instruments may capture the patient's perception of the disease. In this context, a similarity between PASS and patient-perceived low disease activity in the REFLAP study can be found..$^{31}$ In conclusion, this study showed that PASS might be used as an alternative for assessment of low disease activity and overall disease status in patients with PsA in real clinical practice.

Correction notice The article has been corrected since it was published online. The co-author Juan D Cañete's name was published incorrectly which has been amended now.

Acknowledgements We want to thank all the study participants.

Contributors All listed authors equally contributed to planning, conduct, collection of data and writing of the work.

Funding The authors have not declared a specific grant for this research from any funding agency in the public, commercial or not-for-profit sectors.

Competing interests None declared.

\section{Patient consent for publication Not required.}

Ethics approval The study protocol was in compliance with the Declaration of Helsinki, and written consent was obtained from each participant. The study was approved by the Institutional Review Board of the University of Molise (protocol $\mathrm{n}$. 0001-09-2017)

Provenance and peer review Not commissioned; externally peer reviewed. Data availability statement Data are available upon reasonable request.

Open access This is an open access article distributed in accordance with the Creative Commons Attribution Non Commercial (CC BY-NC 4.0) license, which permits others to distribute, remix, adapt, build upon this work non-commercially, and license their derivative works on different terms, provided the original work is properly cited, appropriate credit is given, any changes made indicated, and the use is non-commercial. See: http://creativecommons.org/licenses/by-nc/4.0/.

ORCID iD

Ennio Lubrano http://orcid.org/0000-0001-6189-5328

\section{REFERENCES}

1 Lubrano E, Scriffignano S, Perrotta FM. Psoriatic arthritis, psoriatic disease, or psoriatic syndrome? J Rheumatol 2019;46:1428-30.

2 Perrotta FM, Marchesoni A, Lubrano E. Minimal disease activity and remission in psoriatic arthritis patients treated with anti-TNF- $\alpha$ drugs. J Rheumatol 2016;43:350-5.

3 Lubrano E, Parsons WJ, Perrotta FM. Assessment of response to treatment, remission, and minimal disease activity in axial psoriatic arthritis treated with tumor necrosis factor inhibitors. J Rheumatol 2016;43:918-23.

4 Perrotta FM, Lubrano E. Subcutaneous anti-TNF alfa induced sustained minimal disease activity and remission in psoriatic arthritis patients: a retrospective study. Postgrad Med 2016;128:693-6.

5 Smolen JS, Schöls M, Braun J, et al. Treating axial spondyloarthritis and peripheral spondyloarthritis, especially psoriatic arthritis, to target: 2017 update of recommendations by an international Task force. Ann Rheum Dis 2018;77:3-17.

6 Lubrano E, De Socio A, Perrotta FM. Comparison of composite indices tailored for psoriatic arthritis treated with csDMARD and bDMARD: a cross-sectional analysis of a longitudinal cohort. $J$ Rheumatol 2017;44:1159-64.

7 Lubrano E, Scriffignano S, Perrotta FM. Residual disease activity and associated factors in psoriatic arthritis. J Rheumatol 2019. doi:10.3899/jrheum.190679. [Epub ahead of print: 01 Nov 2019].

8 Lubrano E, Perrotta FM. Psoriatic arthritis: is it time to treat-to-target or target to treat? Clin Rheumatol 2017;36:2633-5.

9 Orbai A-M, de Wit M, Mease PJ, et al. Updating the psoriatic arthritis (PSA) core domain set: a report from the PSA workshop at OMERACT 2016. J Rheumatol 2017;44:1522-8.

10 Goncalves R, Martins L, mariz H, et al. MDA Versus DAPSA: Applicability in a Real World [abstract].. Arthritis Rheumatol 2019;71.

11 Lubrano E, Perrotta FM, Parsons WJ, et al. Patient's global assessment as an outcome measure for psoriatic arthritis in clinical practice: a surrogate for measuring low disease activity? $J$ Rheumatol 2015;42:2332-8.

12 Saag KG. OMERACT 6 brings new perspectives to rheumatology measurement research. J Rheumatol 2003;30:639-41.

13 Maksymowych WP, Gooch K, Dougados M, et al. Thresholds of patient-reported outcomes that define the patient acceptable symptom state in ankylosing spondylitis vary over time and by treatment and patient characteristics. Arthritis Care Res 2010;62:826-34.

14 Dougados M, Luo MP, Maksymowych WP, et al. Evaluation of the patient acceptable symptom state as an outcome measure in patients with ankylosing spondylitis: data from a randomized controlled trial. Arthritis Rheum 2008;59:553-60.

15 Tubach F, Ravaud P, Baron G, et al. Evaluation of clinically relevant states in patient reported outcomes in knee and hip osteoarthritis: the patient acceptable symptom state. Ann Rheum Dis 2005;64:34-7.

16 Heiberg T, Kvien TK, Mowinckel P, et al. Identification of disease activity and health status cut-off points for the symptom state acceptable to patients with rheumatoid arthritis. Ann Rheum Dis 2008;67:967-71.

17 Kvamme MK, Kristiansen IS, Lie E, et al. Identification of cutpoints for acceptable health status and important improvement in patientreported outcomes, in rheumatoid arthritis, psoriatic arthritis, and ankylosing spondylitis. J Rheumatol 2010;37:26-31. 
18 Tubach F, Ravaud P, Martin-Mola E, et al. Minimum clinically important improvement and patient acceptable symptom state in pain and function in rheumatoid arthritis, ankylosing spondylitis, chronic back pain, hand osteoarthritis, and hip and knee osteoarthritis: results from a prospective multina. Arthritis Care Res 2012;64:1699-707.

19 Fei JZ, Perruccio AV, JY Y, et al. The relationship between patient acceptable symptom state and disease activity in patients with psoriatic arthritis. Rheumatology 2019

20 Schoels MM, Aletaha D, Alasti F, et al. Disease activity in psoriatic arthritis (PSA): defining remission and treatment success using the DAPSA score. Ann Rheum Dis 2016;75:811-8.

21 Coates LC, Fransen J, Helliwell PS. Defining minimal disease activity in psoriatic arthritis: a proposed objective target for treatment. Ann Rheum Dis 2010;69:48-53.

22 Coates LC, Helliwell PS. Defining low disease activity states in psoriatic arthritis using novel composite disease instruments. $J$ Rheumatol 2016;43:371-5.

23 Gossec L, de Wit M, Kiltz U, et al. A patient-derived and patientreported outcome measure for assessing psoriatic arthritis: elaboration and preliminary validation of the psoriatic arthritis impact of disease (PsAID) questionnaire, a 13-country EULAR initiative. Ann Rheum Dis 2014;73:1012-9.

24 Ranza R, Marchesoni A, Calori G, et al. The Italian version of the functional disability index of the health assessment questionnaire.
A reliable instrument for multicenter studies on rheumatoid arthritis. Clin Exp Rheumatol 1993;11:123-8.

25 Taylor W, Gladman D, Helliwell P, et al. Classification criteria for psoriatic arthritis: development of new criteria from a large international study. Arthritis Rheum 2006;54:2665-73.

26 Healy PJ, Helliwell PS. Measuring clinical enthesitis in psoriatic arthritis: assessment of existing measures and development of an instrument specific to psoriatic arthritis. Arthritis Rheum 2008;59:686-91.

27 Lubrano E, Perrotta FM, Scriffignano S, et al. Sustained very low disease activity and remission in psoriatic arthritis patients. Rheumatol Ther 2019;6:521-8.

$28 \mathrm{McHugh}$ ML. Interrater reliability: the kappa statistic. Biochem Med 2012;22:276-82.

29 Solomon DH, Bitton A, Katz JN, et al. Review: treat to target in rheumatoid arthritis: fact, fiction, or hypothesis? Arthritis Rheumatol 2014;66:775-82.

30 Conti F, Ceccarelli F, Massaro L, et al. Evaluation of the patient acceptable symptom state (pass) in Italian patients affected by systemic lupus erythematosus: association with disease activity indices. PLoS One 2013;8:e73517.

31 Gorlier C, Orbai A-M, Puyraimond-Zemmour D, et al. Comparing patient-perceived and physician-perceived remission and low disease activity in psoriatic arthritis: an analysis of 410 patients from 14 countries. Ann Rheum Dis 2019;78:201-8. 